\title{
PREDICTORES DE LA IDEACIÓN SUICIDA: UN ESTUDIO EMPÍRICO EN ADOLESCENTES
}

\author{
NURIA FERNÁNDEZ e HIPÓLITO MERINO \\ Universidad de Santiago de Compostela
}

(Aceptado en junio de 2001)

\begin{abstract}
Últimamente ha habido un aumento de los fenómenos suicidas adolescentes. En este trabajo se pretenden estudiar los índices de ideación suicida adolescente y la capacidad del autoconcepto y de la depresión para predecir los pensamientos de suicido, teniendo en cuenta las posibles diferencias asociadas al sexo. Para ello se aplicaron, a 161 adolescentes de 16 a 19 años escolarizados en BUP, COU y FP de segundo ciclo, la escala de ideación suicida (Beck, 1979), adaptada y abreviada por Villardón (1993), la escala de autoconcepto (Villa, 1992) y el inventario de depresión de Beck (BDI) adaptado por Vázquez y Sanz. Los resultados indicaron que las chicas tenían niveles más altos de ideación suicida que los chicos y que mientras en el grupo de las chicas la depresión explicaba hasta un $64 \%$ de varianza de ideación suicida en el grupo de los chicos la dimensión social de autoconcepto era la variable que explicaba mayor proporción de varianza de la variable criterio. Las diferencias observadas entre ambos sexos señalan la conveniencia de la aproximación de género para incidir en la ideación suicida en la población adolescente.
\end{abstract}

Palabras clave: Ideación suicida, autoconcepto, depresión, adolescencia.

\section{Predictors of suicidal thinking: an empirical study of adolescents}

The rate of suicide and attempted suicide among adolescents is rising. In this work we studied the degree of suicidal thinking among adolescents, and the capacity of measures of self-concept and depression to predict suicidal thinking, related to possible sex differences. A hundred and sixty-one adolescents aged 16-19 years who were attending school or vocational training centres were administered the Suicidal Ideation Scale (SIS; Beck, 1979) adapted for the Spanish population and abbreviated by Villardon (1993), the Self-Concept Scale (SCS; Villa, 1992), and the Beck Depression Inventory (BDI) adapted for the Spanish population by Vázquez and Senz. Results showed that girls had higher SIS scores than boys, and whereas $64 \%$ of the variance of the girls'SIS scores was accounted for by their BDI scores, the predictor accounting for most variance in the boys'SIS scores was the SCS score. This work highlight the need for interventions designed to prevent suicidal thinking among adolescentes to take gender into account.

Key words: Suicidal thinking, self-concept, depression, adolescence.

\section{INTRODUCCIÓN}

En los últimos años ha habido un aumento de los fenómenos suicidas en los adolescentes (De Wilde, Kienhorst, y Diekstra, 1996; González, Berenzon, y Jiménez, 1999). El suicidio se ha convertido, así, en la segunda causa más frecuente de

Correspondencia: Hipolito Merino, Departamento de Psicología Clínica y Psicobiología, Facultad de Psicología, Universidad de Santiago de Compostela, Campus Universitario Sur, 15706. Teléfono: 981563100 , Ext.13742. Correo-e: hmerino@usc.es muerte en numerosos países, entre ellos España (Alonso-Fernández, 1985). Pese a ello, la ideación suicida ha sido poco estudiada, y esto limita las posibilidades de desarrollar estrategias eficaces para prevenir tanto intentos de suicidio como suicidios consumados. Los fenómenos suicidas ocurren en un continuum de gravedad creciente que va desde la ideación suicida, el fenómeno suicida menos grave -aunque, por otro lado, el más prevalente-, a la consumación del sui- 
cidio. La ideación suicida, los intentos de suicidio y el suicidio consumado son conductas que están relacionadas jerárquicamente: los pensamientos suicidas generalmente preceden a los actos suicidas y muchos suicidios consumados están precedidos de intentos de suicidio (De Wilde et al., 1996).

Investigaciones comunitarias en poblaciones adolescentes han señalado la existencia de una gran variedad de resultados en los datos de prevalencia de ideación suicida. Dicha variedad oscila entre un 3,5 por 100 y un 52,9 por 100 (Harkavy, Asnis, Boeck, y DiFiore, 1987). En gran medida, las diferencias en las tasas de ideación suicida pueden ser explicadas por la disparidad que existe entre los distintos estudios en los períodos de referencia y en el tipo de instrumentos de evaluación, así como en la noción de ideación suicida.

La ideación suicida se refiere a cogniciones que pueden variar desde pensamientos fugaces de que la vida no vale la pena vivirla, hasta imágenes autodestructivas persistentes y recurrentes (Goldney, Winefield, Tiggemann, Winefield $y$ Smith, 1989). El período de referencia varía de unos estudios a otros (Diekstra, 1995). En algunos estudios se pregunta sobre pensamientos de suicidio durante la última semana (Kienhorst, De Wilde, Van de Bout, Diekstra, Wolters, 1990), en otros durante el último año (Dubow , Kausch, Blum y Reed, 1989) y en otros durante toda la vida (Harkavy et al.,1987). Los estudios con períodos de referencia largos obtienen tasas de ideación suicida más altas que los estudios con períodos de referencia cortos. El tipo de instrumentos de evaluación también varía de unos estudios a otros. Mediante los cuestionarios autoaplicados se obtienen tasas de ideación suicida más elevadas que con las entrevistas (Klimes-Dougan, 1998).

La delimitación de los factores capaces de determinar una mayor vulnerabilidad para el desarrollo de intentos de suicidio y suicidios consumados, ha sido una de las líneas de investigación que ha alcanzado mayor relieve en los últimos años en el estudio de los fenómenos suicidas (Buendía, Riquelme y Ruiz, 1996). Sin embargo, todavía existen pocos estudios que aborden las características que distinguen a los escolares adolescentes con ideación suicida, existiendo así, un importante desconocimiento sobre el peso específico que cada una de estas características puede tener en la ideación suicida por separado, y lo que es más importante, respecto a las interacciones que pueden mantener entre sí como indicadores de vulnerabilidad para el desarrollo de conductas suicidas en este grupo de población. Los estudios existentes han señalado que la ideación suicida en adolescentes está relacionada con variables tales como la edad (De Man, Leduc y Labrèche Gauthier, 1993); el sexo (Charron, 1981; Côté, Pronovost y Ross, 1989); baja autoestima (Bagley, 1975; Cole, Protinsky y Cross, 1993; Robbins y Alessi, 1985); locus de control externo (Budner y Kumler, 1973; Topol y Reznikoff, 1982); estrés negativo (De Man, Leduc y Labrèche-Gauthier, 1993; Dubow et al., 1989); afrontamiento no adaptativo (Villardón, 1993); consumo de alcohol y drogas (Corbeil, 1984; Dukes y Lorch, 1989); falta de apoyo social (Bagley, 1975; KirkpatrickSmith, Rich, Bonner y Jans, 1989); depresión (De Man, 1999; Goldberg, 1981; Lester y Gatto, 1989; Zhang, 1996) y desesperanza (Villardón, 1993).

El autoconcepto y la ideación suicida están muy relacionados. Esta asociación todavía es mayor en la adolescencia, al ser ésta una etapa de transición y cambio en la que el sujeto debe adaptarse a una serie de modificaciones de su imagen física y de su realidad social e integrar en su autoconcepto esta nueva realidad de su identidad. A este respecto, Pinto y Whisman (1996) y Siemen, Warrington y 
Mangano (1994) señalaron que los adolescentes con alta ideación suicida presentaban peor autoconcepto que los sujetos controles.

Por otro lado, la depresión también se ha relacionado con la ideación suicida adolescente. En algunos estudios la ideación suicida se considera como síntoma o como consecuencia de dichos trastornos depresivos (Goldberg, 1981). En otros estudios, en cambio, la depresión aparece como un factor que influye en la aparición de la ideación suicida, y que puede ver incrementada o disminuida su accion por la presencia de otros factores. Estos estudios, por tanto, señalan una conexión entre depresión e ideación suicida, pero no una correspondencia exacta. Ni todos los depresivos presentan ideación suicida, ni todos los que presentan ideación suicida son depresivos. Desde este enfoque, la ideación suicida es, por tanto, un fenómeno multidimensional en el que intervienen gran variedad de factores que interactúan entre sí. Dentro de este grupo de estudios, Villardón (1993) y De Man (1999) señalaron que la depresión era la variable que explicaba mayor proporción de varianza de ideación suicida.

Mediante este trabajo se pretenden estudiar los índices de ideación suicida adolescente y la capacidad del autoconcepto y la depresión para predecir los pensamientos de suicidio, teniendo en cuenta las diferencias asociadas al sexo.

\section{MÉTODO}

\section{Sujetos}

La muestra consta de 161 adolescentes gallegos escolarizados en $3^{\circ}$ de BUP-COU (107) y FP de segundo ciclo (54) en dos centros de enseñanzas medias públicos en el curso 1999-2000. De los 161 ado- lescentes, 71 son varones y 90 mujeres. La edad de la muestra oscila entre los 16 y los 19 años. (Los cuestionarios de los alumnos que tenían más de 19 años fueron eliminados).

\section{Instrumentos}

Al encuestado se le preguntó la edad, el sexo y el tipo de estudios que estaba cursando. Además, se administraron los siguientes instrumentos: a) la escala de autoconcepto de Villa (1992); b) la escala de depresión de Beck, versión española adaptada por Vázquez y Sanz (1991); c) y la escala de ideación suicida de Beck, en su versión adaptada y abreviada por Villardón (1993). El contenido y las características de estos instrumentos se exponen a continuación.

La escala de autoconcepto de Villa se compone de 26 items. Cada uno de ellos tiene cinco opciones de respuesta (de muy de acuerdo a muy en desacuerdo). El análisis factorial de componentes principales y rotación varimax dio como resultado tres factores interpretables en la escala de autoconcepto: autoestima, dimensión social de autoconcepto y autoconcepto académico. Los indices de fiabilidad de los tres factores (alpha de Cronbach) oscilan entre 0,81 (autoconcepto académico) y 0,92 (autoestima).

La escala autoaplicada de depresión de Beck adaptada por Vázquez y Sanz (1991) consta de 21 items que evalúan la intensidad del síndrome depresivo. Los autores obtuvieron un coeficiente de fiabilidad test-retest de 0,65 a 0,72 y un alfa de Cronbach de 0,82. •

La escala de ideación suicida de Beck adaptada y abreviada por Villardón consta de 10 items con cuatro opciones de respuesta. La consistencia interna de la escala hallada a través de alpha de Cronbach es muy alta $(0,90)$. 


\section{Procedimiento}

Los cuestionarios se aplicaron de forma colectiva en las aulas de $3^{2}$ de BUP, COU y FP de segundo ciclo en dos centros de enseñanzas medias en el transcurso de una clase de 50 minutos, previa autorización de la dirección de los centros. Se solicitó la participación voluntaria de los estudiantes explicando que se estaba realizando un estudio para conocer el estado de bienestar/malestar psicológico de los adolescentes. Dicha participación fue requerida garantizando el anonimato y confidencialidad en el manejo de la información.

\section{Análisis de datos}

Los datos de los 161 adolescentes que participaron en la investigación han sido tratados mediante el paquete estadístico SPSS. Los análisis abarcan descriptivos básicos, análisis de comparación entre medias (" $t$ " de Student y análisis de varianza) y análisis de regresión múltiple.

\section{RESULTADOS}

Un $10,5 \%$ de los adolescentes objeto de estudio presenta ideación suicida elevada. Para calcular este índice, se calculó, siguiendo a Villardón, el porcentaje de sujetos que respondió las opciones 3 y 4 en cinco o más items de la escala de ideación suicida.
En la Tabla 1 se presentan las puntuaciones medias y las desviaciones típicas de las dimensiones de autoconcepto, depresión e ideación suicida para la muestra total, así como para el grupo de los chicos y el de las chicas. Por otro lado, también se especifica el contraste de las diferencias entre chicos y chicas en las dimensiones de autoconcepto, depresión e ideación suicida así como la significación de dichas diferencias entre ambos sexos.

En todas las variables que intervienen en el estudio - menos en la dimensión de autoconcepto- se encuentran diferencias significativas asociadas al sexo. La ideación suicida es la variable en la que se obtienen mayores diferencias entre el grupo de los chicos y el de las chicas. Al considerar la interacción sexo y tipo de estudios se obtienen las puntuaciones medias de: 14,48 para los chicos de BUP-COU, 12,17 para los chicos de FP, 16,94 para las chicas de BUP-COU y 20,25 para las chicas de FP. El efecto de la interacción sexo y tipo de estudios es estadísticamente significativa $(F=7,535$, $p<0,05)$.

Se realizaron análisis de regresión múltiple (stepwise) para obtener información sobre el poder que tenían las dimensiones de autoconcepto y la depresión para explicar la ideación suicida. Los análisis se realizaron considerando la muestra general y los grupos de chicos y chicas separadamente.

Tabla 1. Datos descriptivos y resultados de los análisis de varianza tomando el sexo como variable de agrupación

\begin{tabular}{lcccccccc}
\hline & \multicolumn{2}{c}{ Muestra Total } & \multicolumn{2}{c}{ Chicos } & \multicolumn{2}{c}{ Chicas } \\
\cline { 2 - 9 } & $M$ & $D T$ & $M$ & $D T$ & $M$ & $D T$ & $T$ & Sig. \\
\hline Autoc.académico. & $\mathbf{1 6 , 3 8 0}$ & $\mathbf{3 , 6 6 8}$ & 16,377 & $\mathbf{3 , 5 9 8}$ & $\mathbf{1 6 , 3 8 2}$ & $\mathbf{3 , 7 4 3}$ & $-\mathbf{0 , 0 0 9}$ & 0,993 \\
Autoestima & $\mathbf{4 5 , 2 4 2}$ & $\mathbf{9 , 2 8 0}$ & $\mathbf{4 8 , 6 5 7}$ & $\mathbf{7 , 5 2 7}$ & $\mathbf{4 2 , 4 9 4}$ & $\mathbf{9 , 6 7 6}$ & $\mathbf{4 , 3 6 9}$ & 0,000 \\
Dim.social autoc. & 35,956 & $\mathbf{6 , 4 9 5}$ & $\mathbf{3 7 , 2 9 4}$ & $\mathbf{6 , 2 5 6}$ & $\mathbf{3 4 , 9 4 4}$ & $\mathbf{6 , 5 2 3}$ & $\mathbf{2 , 2 8 2}$ & 0,024 \\
Depresión & 11,860 & $\mathbf{9 , 3 3 2}$ & $\mathbf{8 , 4 7 0}$ & $\mathbf{7 , 5 8 6}$ & 14,523 & $\mathbf{9 , 7 4 4}$ & $\mathbf{- 4 , 2 7 8}$ & $\mathbf{0 , 0 0 0}$ \\
Ideación Suicida & 15,783 & 6,346 & 13,380 & $\mathbf{4 , 4 7 6}$ & $\mathbf{1 7 , 6 7 8}$ & $\mathbf{6 , 9 5 7}$ & $\mathbf{- 4 , 7 4 6}$ & $\mathbf{0 , 0 0 0}$ \\
\hline
\end{tabular}


Tabla 2. Resultados de los análisis de regresión tomando las dimensiones de autoconcepto y la depresión como predictores y la ideación suicida como criterio.

\begin{tabular}{|c|c|c|c|c|c|c|}
\hline & Pasos & $\begin{array}{c}\text { Variables } \\
\text { introducidas }\end{array}$ & R. Cuadrado & Beta & $T$ & Sig. \\
\hline Total & $\begin{array}{l}1 \\
2\end{array}$ & $\begin{array}{l}\text { Depresión } \\
\text { Autoestima }\end{array}$ & $\begin{array}{l}0,439 \\
0,513\end{array}$ & $\begin{array}{r}0,466 \\
-0,342\end{array}$ & $\begin{array}{r}6,409 \\
-4,709\end{array}$ & $\begin{array}{l}0,000 \\
0,000\end{array}$ \\
\hline Chicos & 1 & $\begin{array}{l}\text { Dim.social } \\
\text { autoconcep. }\end{array}$ & 0,205 & $-0,467$ & $-4,054$ & 0,000 \\
\hline Chicas & 1 & Depresión & 0,637 & 0,801 & 11,808 & 0,000 \\
\hline
\end{tabular}

La depresión y la autoestima explican un $51 \%$ de varianza de ideación suicida en la muestra general. Por otro lado, la dimensión social de autoconcepto y la depresión son las variables que, respectivamente, muestran una mayor capacidad explicativa de la ideación suicida en el grupo de los chicos y de las chicas.

\section{DISCUSIÓN}

Un porcentaje considerable de adolescentes $(10,5 \%)$ piensa en el suicidio de una forma seria, frecuente y continuada. Este dato muestra un alto nivel de concordancia con las proporciones obtenidas por Medina, López, Villatoro, Juárez, Carreño, Berenzon y Rojas (1994) y Villardón (1993). Los primeros encontraron que el $10 \%$ de los adolescentes del Distrito Federal de México presentaba todos los indicadores de ideación suicida. El segundo, a su vez, halló que el $10,3 \%$ de los escolares vizcaínos de 14 a 17 años tenía elevada ideación suicida.

Afortunadamente, la relación entre ideación suicida y realización de actos autodestructivos no es, en absoluto, directa ni total: se ha señalado que un $20 \%$ de los adolescentes que piensa frecuentemente en suicidio acaba por realizar, finalmente, un intento suicida (Davidson y Philippe, 1986; en Fernández Rivas, 1995). Sin embargo, pese a que tener pensamientos suicidas no implique la ejecución de conductas suicidas, resulta prioritario detectar adolescentes con pensamientos suicidas y orientar esfuerzos preventivos entre esta población, dada la fuerte asociación existente en la adolescencia entre ideación suicida y conducta suicida no fatal $\mathrm{y}$, más aún, entre ideación suicida y conducta suicida múltiple (González Forteza, 1998; Kienhorst, et al., 1990).

En nuestro estudio, las mujeres tienen niveles significativamente más altos de ideación suicida que los varones. Las diferencias entre ambos sexos son más acusadas en los estudiantes de FP que en los de BUP-COU. Estos datos son coincidentes con lo que aparece en la literatura referido a la mayor prevalencia de ideación suicida en las adolescentes que en los adolescentes (Medina Mora et al., 1994; Villardón, 1993).

Las diferencias de género también se observan en las variables predictoras de la ideación suicida. En los chicos, la variable que explica mayor proporción de varianza de ideación suicida es la dimensión social de autoconcepto. En las chicas, en cambio, es la depresión.

Por tanto, la ideación suicida parece requerir de distintos acercamientos en los adolescentes y en las adolescentes. Las diferencias observadas en pensamiento de suicidio entre ambos sexos sugieren la conveniencia de tener en cuenta el género a la hora de prevenir e intervenir la ideación suicida en varones y mujeres adolescentes. 


\section{REFERENCIAS BIBLIOGRAFIA}

Alonso-Fernández, F. (1985). Conducta suicida en la adolescencia (un ensayo de penetración comprensiva en las estadísticas). Psicopatología, 5, 147-159.

Bagley, C. (1975). Suicidal behaviour and suicidal ideation in adolescents. British Journal of Guidance \& Counselling, 3, 190-208.

Budner, S., y Klumer, F. (1973). Correlates of suicidal ideation: Houston: American Association of Suicidology.

Buendía, J., Riquelme, A., y Ruiz, J.A. (1996). Estrés y suicidio en la adolescencia. En J. Buendía (Ed.), Psicopatología en niños y adolescentes. Madrid: Pirámide.

Charron, M.F. (1981). Le suicide au Québec, analyse statisque. Québec, Canada: Ministère des Affaires Sociales.

Cole, D.E., Protinsky, H.O., y Cross, L.H. (1993). An empirical investigation of adolescent suicidal ideation. Adolescence, 27, 813-818.

Corbeil, S. (1984). Suicide et adolescence (Suicide and adolescence). En P. Morisette (Ed), Le suicide, démystification, intervention, prévention (pp. 272-306). Québec, Canada: Garotex.

Côté, L., Pronovost, J., y Ross, C. (1989). Etude sur la prévalence des comportements suicidaires chez les adolescents de la régimen 04: Faits saillants . Shawinigan, Québec, Canada: Département de santé communautaire.

De Man, A.F. (1999). Correlates of suicide ideation in high school students: the importance of depression. The Journal of Genetic Psychology, 160, 105-114.

De Man, A.F., Leduc, C.P., y Labrèche-Gauthier, L. (1992). Correlates of suicide ideation in french-canadian adults and adolescents: a comparison. Journal of Clinical Psychology, 48, 811-816.

De Man, A. F., Leduc, C.P., y Labréche-Gauthier, L. (1993). Correlates of suicidal ideation in french-canadian adolescents: personal variables, stress, and social support. Adolescence, 28, 819-830.

De Wilde, E.J., Kienhorst, I.C., y Diekstra, R.F.W. (1996). El suicidio en la adolescencia. En J. Buendía (Ed.), Psicopatología en niños y adolescentes. Madrid: Pirámide.
Diekstra, R.F.W. (1995). Depression and suicidal behaviors in adolescence: sociocultural and time trends. En M. Rutter y D.J. Smith (Eds.), Psychosocial disorders in young people: time trends and their causes. Chichester: Wiley.

Dubow, E.F., Kausch, D.F., Blum, M.C., y Reed, J. (1989). Correlates of suicidal ideation and attempts in a community sample of junior high and high school students. Journal of Clinical Child Psychology, 18, 158-166.

Dukes, R.L., y Lorch, B.D.(1989). The effects of school, family, self-concept, and deviant behavior on adolescent suicide ideation. Journal of Adolescence, 12, 239-251.

Goldberg, E.L. (1981). Depression and suicide ideation in the young adult. American Journal of Psychiatry, 138, 35-40.

Goldney, R.D., Winefield, A.H., Tiggemann, M., Winefield, H.R., y Smith, S. (1989). Suicidal ideation in a young adult population. Acta Psychiatrica Scandinavica, 79, 481-489.

González, C., Berenzon, S., y Jiménez, A. (1999). Al borde de la muerte: problemática suicida en adolescentes. Salud Mental, Número Especial.

González, C., Berenzon, S., Tello, A.M., Facio, D., y Medina (1998). Ideación suicida y características asociadas en mujeres adolescentes. Salud Pública de México, 40, 5.

Harkavy, J.M., Asnis, G.M., Boeck, M., y DiFiore, J. (1987). Prevalence of specific suicidal behaviors in a high school sample, American Journal of Psychiatry, 144, 12031206.

Kienhorst, C., De Wilde, E., Van de Bout, J., Dieskstra, R., y Wolters, W. (1990). Characteristics of suicide attempters in a population-based sample of Dutch adolescents. British Journal Psychiatry, 156, 234-248.

Kirkpatrick-Smith, K., Rich, A., Bonner, R., y Jans, F. (1989). Substance abuse and suicidal ideation among adolescents. En D. Lester (Ed.), Suicide '89 (pp. 90-91). Denver, CO: AAS.

Klimes-Dougan, B. (1998). Screening for suicidal ideation in children and adolescents: methodological considerations. Journal of Adolescence, 21, 435-444. 
Lester, D., y Gatto, J. (1989). Self-destructive tendencies and depression as predictors of suicidal ideation in teenagers. Journal of Adolescence, 12, 221-223.

Medina, M.A., López, E.K., Villatoro, J.A., Juárez, F., Carreño, S., Berenzon, S., y Rojas, E. (1994). La relación entre la ideación suicida y el abuso de sustancias. Resultados de una encuesta en la población estudiantil. Anales del Instituto Mexicano de Psiquiatría, Reseña de la IX Reunión de Investigación, 7, 14, 1994.

Pinto, A., y Whisman, M.A. (1996). Negative affect and cognitive biases in suicidal and nonsuicidal hospitalized adolescents. Journal of the American Academy of Child and Adolescent Psychiatry, 35, 2, 158-165.

Robbins, D.R., y Alessi, N.E. (1985). Depressive symptoms and suicidal behavior in adolescents. American Journal of Psychiatry, 142, 588-592.

Siemen, J.R., Warrington, C.A., y Mangano, E.L. (1994). Comparison of the Millon Adolescent Personality Inventory and the Suicide Ideation Questionnaire-Junior with an adolescent inpatient sample. Psychological Reports, 75, 2, 947-950.

Topol, P., y Reznikoff, M. (1982). Perceived peer and family relationships, hopelessness and locus of control as factors in adolescent suicide attempts. Suicide and LifeThreatening Behavior, 12, 141-150.

Villardón, L. (1993). El pensamiento de suicidio en la adolescencia. Bilbao: Universidad de Deusto.

Zhang, J. (1996). Suicide in Beijing, China, 1992-1993. Suicide and Life-Threatening Behavior, 26, 175-180. 